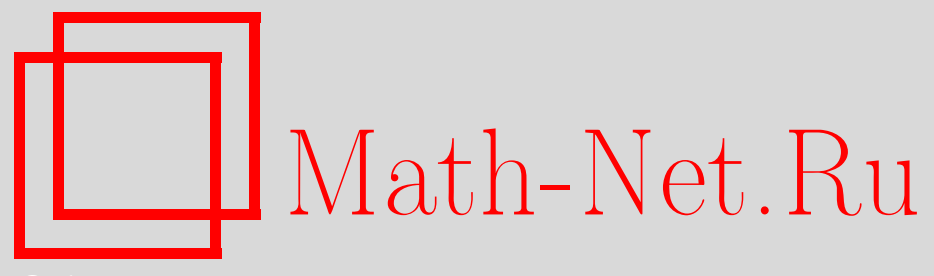

А. И. Есина, А. И. Шафаревич, Условия квантования на римановых поверхностях и квазиклассический спектр оператора Шрёдингера с комплексным потенциалом, Матем. заметки, 2010, том 88, выпуск 2, 229-248

DOI: https://doi.org/10.4213/mzm8803

Использование Общероссийского математического портала Math-Net.Ru подразумевает, что вы прочитали и согласны с пользовательским соглашением http: //www . mathnet.ru/rus/agreement

Параметры загрузки:

IP : 54.81 .137 .203

26 апреля 2023 г., 17:04:04

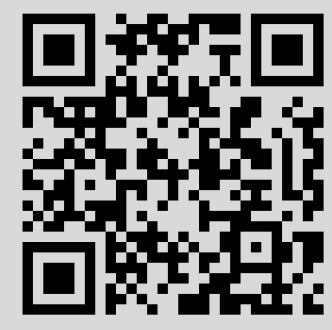


Том 88 выпуск 2 август 2010

УДК $517.984 .55+514.84$

\section{Условия квантования на римановых поверхностях и квазиклассический спектр оператора Шрёдингера с комплексным потенциалом}

\section{А. И. Есина, А. И. Шафаревич}

Описана асимптотика спектра оператора

$$
\widehat{H}\left(x,-\imath h \frac{\partial}{\partial x}\right)=-h^{2} \frac{\partial^{2}}{\partial x^{2}}+\imath(\cos x+\cos 2 x)
$$

при $h \rightarrow 0$. Показано, что спектр концентрируется вблизи некоторого графа на комплексной плоскости. Получены уравнения на собственные значения, которые представляют собой условия на периоды голоморфной формы на соответствующей римановой поверхности.

Библиография: 15 названий.

1. Введение. Одно из центральных приложений теории квазиклассического квантования (см., например, [1]) - описание асимптотики спектра операторов вида $\widehat{H}(x,-\imath h \partial / \partial x)$ при $h \rightarrow 0$. При этом задачу естественно разделить на две:

1) приближенное решение спектрального уравнения, т.е. нахождение чисел $E$ и функций $\Psi$, удовлетворяющих равенству

$$
\widehat{H} \Psi=E \Psi+O\left(h^{N}\right)
$$

2) выбор среди чисел $E$ таких, которые приближают точки спектра оператора $\widehat{H}$, т.е. таких, что

$$
|E-\lambda|=O\left(h^{N}\right)
$$

для некоторой точки $\lambda$ из спектра $\widehat{H}$.

Если оператор $\widehat{H}$ самосопряжен, оценка (1.2) автоматически вытекает из равенства (1.1) (см., например [1], [2]); в то же время, задача 1) весьма нетривиальна и связана с изучением инвариантных множеств соответствующей классической гамильтоновой системы. Несамосопряженный случай исследован гораздо менее полно; вместе с тем, спектральные задачи для несамосопряженных операторов возникают во многих важных физических приложениях (теория гидродинамической устойчивости, описание магнитных полей Земли и галактик и др. (см., например, [3], [4])).

Работа выполнена при поддержке Российского фонда фундаментальных исследований (гранты №oo 08-01-00726a, 07-01-00648a) и Министерства образования (гранты №№ РНП 2.1.1.3704, МОН 2.1.1.4540).

(С) А.И. ЕСинА, А.И. ШАФАРЕвич, 2010 
В этой работе рассматривается уравнение Шрёдингера:

$$
-h^{2} \psi^{\prime \prime}+\imath V(x) \psi=E \psi
$$

где $h$ - малый параметр, $V(x)=\cos x+\cos 2 x$, с условием периодичности функции $\psi$, т.е. спектральная задача для оператора

$$
\widehat{H}\left(x,-\imath h \frac{\partial}{\partial x}\right)=-h^{2} \frac{\partial^{2}}{\partial x^{2}}+\imath(\cos x+\cos 2 x)
$$

на окружности. Аналогичные задачи для операторов на отрезке с потенциалами $\imath x$, $\imath x^{2}$ и на окружности с потенциалом $\imath \cos x$ исследовались ранее в работах [5]-[12]. Мы находим асимптотику спектра при $h \rightarrow 0$; при этом оказывается, что числа $E$, удовлетворяющие (1.1), целиком заполняют полуполосу на комплексной плоскости, в то время как настоящий спектр концентрируется вблизи некоторого графа. Основная трудность связана именно с выбором чисел, удовлетворяющих оценке (1.2); этот выбор приводит к условиям квантования на римановой поверхности постоянной комплексной энергии. При описании асимптотики используется техника линий Стокса [14], [15].

2. Формулировка результатов. Основной результат работы - следующее утверждение.

Tеорема 1. 1. Спектр оператора $\widehat{H}$ сосредоточен в $O\left(h^{2}\right)$-окрестности конечного числа аналитических кривых на комплексной плоскости E, которые в совокупности образуют спектральный граф (см. рис. 1).

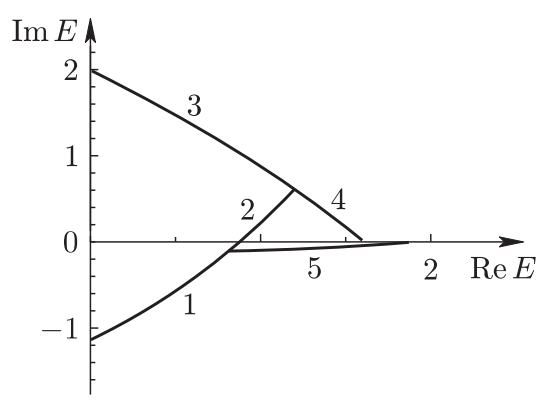

Рис. 1. Спектральный граф

2. Точки спектра находятся в $O\left(h^{2}\right)$-окрестностях решений уравнений

$$
\int_{x_{j}}^{x_{i}} \sqrt{\imath V(x)-E} d x=\imath \pi h\left(n_{i j}+\frac{\gamma}{2}\right),
$$

где $n_{i j}$ - целые числа, $\gamma=0,1, x_{i}$ и $x_{j}$ - нули подынтегрального выражсения.

3. Ребра спектрального графа определяются уравнениями

$$
\operatorname{Re} \int_{x_{j}}^{x_{i}} \sqrt{\imath V(x)-E} d x=0 .
$$

Доказательство теоремы приведено в пп. 6-9. 
3. Псевдоспектр. Как уже отмечалось, числа $E$, удовлетворяющие (1.1), заполняют полуполосу. Такие точки называются точками псевдоспектра оператора $\widehat{H}$. Напомним соответствующее определение [2], [13].

Пусть $A(\varepsilon)$ - семейство операторов в гильбертовом пространстве $H$.

ОпредЕЛЕниЕ 1 . Точка $E$ принадлежит $\varepsilon$-nсевдоспектру оператора $A$, если существует $\psi \in H$ такое, что

$$
\|\psi\|=1, \quad A \psi=E \psi+O(\varepsilon) .
$$

В работе [6] доказано следующее утверждение.

Теорема 2. Для любого $N h^{N}$-nсевдоспектр оператора (1.3) совпадает с полуполосой

$$
[0,+\infty)+\imath[\min V, \max V] .
$$

ЗАмечаниЕ 1. Псевдоспектр совпадает с числовым образом оператора $\widehat{H}$, т.е. с множеством значений квадратичной формы $(\psi, \widehat{H} \psi),\|\psi\|=1$.

4. Матрицы монодромии и уравнения на точки спектра. Для описания асимптотики спектра мы исследуем матрицу монодромии уравнения (1.3), т.е. матрицу оператора сдвига на период в пространстве решений.

ЛЕмма 1 [6]. Условие принадлежности Е дискретному спектру эквивалентно условию $\operatorname{tr} M=\operatorname{det} M+1$, где $M-$ это матрица монодромии.

ДокАЗАТЕЛЬСтво вытекает из того факта, что наличие нетривиального периодического решения эквивалентно наличию собственной функции у оператора монодромии с собственным значением равным 1 и, кроме того, детерминант матрицы монодромии равен 1.

Ниже вычислена асимптотика матрицы монодромии в специальном базисе.

5. Описание техники линий Стокса. Основной инструмент доказательства теоремы 1 - построение ВКБ-асимптотик решений уравнения (1.3) в комплексной области при $h \rightarrow 0$. Для этой цели мы применяем технику линий Стокса [14], [15].

Напомним необходимые определения и утверждения.

ОПРЕДЕЛЕНИЕ 2. 1. Точками поворота называются решения уравнения $E=$ $\imath V(x)$.

2. Линией Стокса называется линия, выходящая из точки поворота $z_{0}$, вдоль которой

$$
\operatorname{Im} \int_{z_{0}}^{z} \sqrt{E-\imath V(x)} d x=0 .
$$

Если точка поворота простая, т.е. $V^{\prime}\left(z_{0}\right) \neq 0$, то из этой точки поворота выходят три линии Стокса под одинаковыми углами в $120^{\circ}$.

3. Область $D$ плоскости $z$ называется канонической, если она ограничена линиями Стокса, содержит внутри себя только одну линию Стокса и отображается функцией

$$
\xi(z)=\int_{z_{0}}^{z} \sqrt{E-\imath V(x)} d x
$$

на всю плоскость с конечным или бесконечным числом вертикальных разрезов. 
В каждой канонической области можно построить ВКБ-асимптотику фундаментальной системы решений уравнения (1.3). Зная взаимное расположение линий Стокса друг относительно друга, можно распространить ВКБ-асимптотику решения уравнения (1.3) из одной канонической области в другую. В результате получаем асимптотику фундаментальной системы решений во всей комплексной плоскости, за исключением малых окрестностей точек поворота.

С каждой тройкой $S, z_{0}, D$ (где $z_{0}$ - точка поворота, $S$ - линия Стокса и $D$ - каноническая область) связана фундаментальная система решений, в данной области имеющая асимптотику

$$
\begin{aligned}
& \psi_{1}=(\imath V(z)-E)^{-1 / 4} \exp \left(\frac{\xi\left(z_{0}, z\right)}{h}\right)(1+O(h)), \\
& \psi_{2}=(\imath V(z)-E)^{-1 / 4} \exp \left(-\frac{\xi\left(z_{0}, z\right)}{h}\right)(1+O(h)),
\end{aligned}
$$

где $\xi\left(z_{0}, z\right)=\int_{z_{0}}^{z} \sqrt{E-\imath V(x)} d x$; ветвь выбирается таким образом, что

$$
\int_{z_{0}}^{z} \sqrt{E-\imath V(x)}: \mathbb{C} \backslash[0,+\infty) \rightarrow\left\{z \in C \mid \operatorname{Re} \int_{z_{0}}^{z} \sqrt{E-\imath V(x)} d x>0\right\} .
$$

ОПРедЕЛЕНИЕ 3. Матрица перехода - это матрица, которая связывает фундаментальные системы решений двух пересекающихся канонических областей.

Если матрицы перехода известны, можно, переходя из одной канонической области в другую, построить матрицу монодромии, которая связывает фундаментальные системы решений в канонических областях $K$ и $K+2 \pi$.

Лемма 2 [14]. Всякая матрица перехода от одной фундаментальной системы решений $к$ другой есть произведение конечного числа элементарных матрии, перехода, принадлежащих к одному из следующих 4 типов.

Tun 1. Переход от элементарной системы решений, определяемой тройкой $\left(S, z_{0}, D_{1}\right)$, к элементарной системе решений, определяемой тройкой $\left(S, z_{0}, D_{2}\right)$. При таком переходе ни линия Стокса, ни направление на ней не меняются; меняется лищь каноническая область, содержащая эту линию Стокса. Матрица перехода выглядит следуюшим образом:

$$
T=\left(\begin{array}{cc}
1+O(h) & 0 \\
0 & 1+O(h)
\end{array}\right)
$$

Tuп 2. Переход от элементарной системы решений, определяемой тройкой $\left(S, z_{0}, D\right), \quad \kappa$ элементарной системе решений, определяемой тройкой $\left(S, z_{1}, D\right)$. $B$ этом случае меняется только направление линии Стокса, такой переход существует только для конечной линии Стокса. Матрица перехода выглядит следующим образом (см. рис. 2):

$$
T=\exp \left(\imath \xi_{0}\right)\left(\begin{array}{cc}
0 & \exp \left(-\frac{\xi\left(z_{1}, z_{2}\right)}{\imath h}\right) \\
\exp \left(\frac{\xi\left(z_{1}, z_{2}\right)}{\imath h}\right) & 0
\end{array}\right),
$$




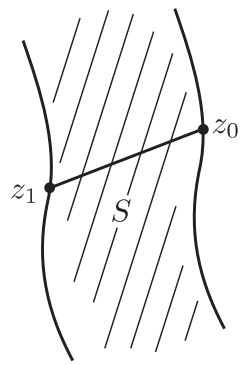

Рис. 2. Иллюстрация ко второй матрице перехода

гдe

$$
\xi_{0}=\lim _{x \rightarrow z_{1}}\left(\arg (\imath V(x)-E)^{-1 / 4}\right)-\lim _{x \rightarrow z_{2}}\left(\arg (\imath V(x)-E)^{-1 / 4}\right) .
$$

Tun 3. Переход от элементарной системы решений, определяемой тройкой $\left(S_{1}, z_{1}, D\right), \kappa$ элементарной системе решений, определяемой тройкой $\left(S_{2}, z_{2}, D\right)$. Причем лучи $\xi\left(S_{1}\right)$ и $\xi\left(S_{2}\right)$ (лежат в $\xi(D)$ ) направлены в одну сторону. Матрица перехода выглядит следующим образом (см. рис. 3 ):

$$
T=\exp \left(\imath \xi_{0}\right)\left(\begin{array}{cc}
\exp \left(-\frac{\xi\left(z_{1}, z_{2}\right)}{\imath h}\right) & 0 \\
0 & \exp \left(\frac{\xi\left(z_{1}, z_{2}\right)}{\imath h}\right)
\end{array}\right)
$$

где

$$
\xi_{0}=\lim _{x \rightarrow z_{1}}\left(\arg (\imath V(x)-E)^{-1 / 4}\right)-\lim _{x \rightarrow z_{2}}\left(\arg (\imath V(x)-E)^{-1 / 4}\right) .
$$

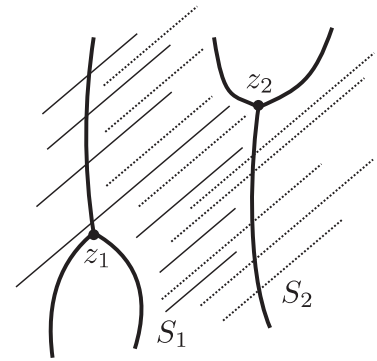

Рис. 3. Иллюстрация к третьей матрице перехода

Tun 4. Переход от элементарной системы решений, определяемой тройкой $\left(S_{1}, z_{0}, D_{1}\right), \kappa$ элементарной системе решений, определяемой тройкой $\left(S_{2}, z_{0}, D_{2}\right)$. Это переход с одной линии Стокса на другую, имеющую то же начало. Матрица перехода выглядит следующим образом (см. рис.4):

$$
T=\exp \left(-\frac{\imath \pi}{6}\right)\left(\begin{array}{cc}
0 & 1 \\
1+O(h) & \imath(1+O(h))
\end{array}\right) .
$$




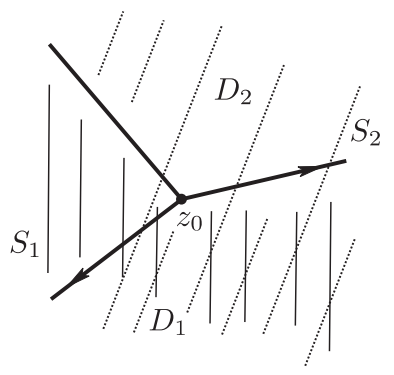

Рис. 4. Иллюстрация к четвертой матрице перехода

6. Асимптотика спектра. В этом пункте мы начинаем доказательство теоремы 1, которому посвящены также пп. 7-9. Сперва опишем точки поворота и различные варианты расположения линий Стокса в нашем случае. Затем выясним, какое расположение линий Стокса дает вклад в спектр, а какое нет. И, наконец, зная расположения линий Стокса, вычислим матрицу монодромии и получим уравнения на точки спектра и на ребра спектрального графа.

Как уже упоминалось, принадлежность $E$ дискретному спектру эквивалентна условию $\operatorname{Tr} M=\operatorname{det} M+1$, где $M-$ матрица монодромии.

7. Точки поворота. При $V(z)=\cos z+\cos 2 z$ на комплексной плоскости существует четыре серии точек поворота, расположенных периодически, с периодом $2 \pi$. Решая уравнение $E=\imath(\cos z+\cos 2 z)$, получаем искомые точки:

$$
\begin{array}{ll}
z_{1}=\arccos \left(\frac{1}{4}(-1+\sqrt{9-8 \imath E})\right), & z_{2}=-\arccos \left(\frac{1}{4}(-1+\sqrt{9-8 \imath E})\right), \\
z_{3}=\arccos \left(\frac{1}{4}(-1-\sqrt{9-8 \imath E})\right), & z_{4}=-\arccos \left(\frac{1}{4}(-1-\sqrt{9-8 \imath E})\right) .
\end{array}
$$

Точки поворота зависят от параметра $E$. Ветвь выбирается следующим образом:

$$
\begin{aligned}
& \arccos (E): \mathbb{C} \backslash[0,+\infty) \rightarrow\{E \in \mathbb{C} \mid \operatorname{Re} \arccos (E)>0\}, \\
& \sqrt{9-8 \imath E}: \mathbb{C} \backslash[0,+\infty) \rightarrow\{E \in \mathbb{C} \mid \operatorname{Re} \sqrt{9-8 \imath E}>0\} .
\end{aligned}
$$

8. Линии Стокса. В зависимости от значения $E$, взаимное расположение линий Стокса может быть различным. Выясним взаимное расположение линий Стокса (Л.С.) в нашем случае при различных $E$ на основе следующих свойств этих линий:

1) Л.С. начинается в точке поворота и заканчивается либо в точке поворота, либо в бесконечности;

2) Л.С. не может содержать точку поворота внутри себя;

3) Л.С. не может пересекать себя или другую Л.С.;

4) из точки поворота порядка $m$ выходят $m+2$ линии Стокса;

5) Л.С. разбивают плоскость $\mathbb{C}$ на области типа полуплоскости и полосы;

$6)$ если $V(x)$ периодичен, то и граф линий Стокса периодичен с тем же периодом $T(T=2 \pi)$;

7) если $V(x)$ - четная функция, то граф линий Стокса симметричен относительно нуля; 
8) граф линий Стокса не может содержать топологическую окружность. Графом линий Стокса называется объединение всех линий Стокса.

ПРЕДЛОЖЕНИЕ 1. В случае $V(z)=\cos z+\cos 2 z$ существует 27 топологически различных случаев взаимного расположения линий Стокса на комплексной плоскости $x$.

ДокАЗАТЕЛЬСтво. Это следует из восьми свойств линий Стокса, приведенных выше, и получается перебором всех возможных случаев. Приведем некоторые из них (см. рис. 5).

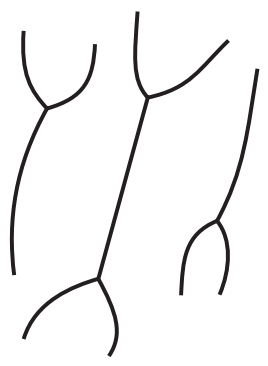

a)

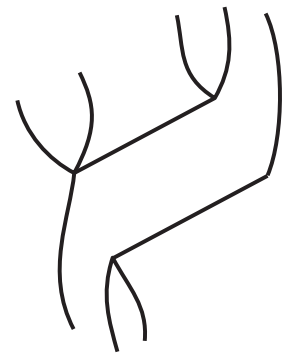

c)

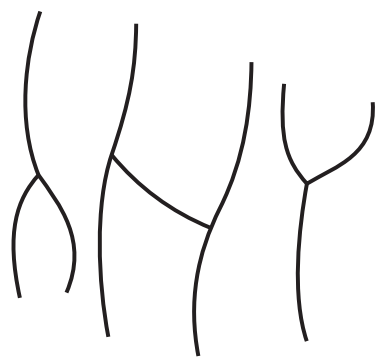

b)

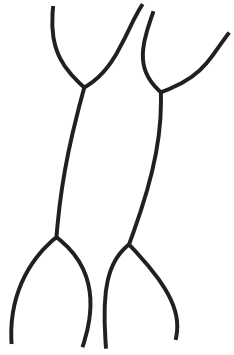

d)

Рис. 5. Некоторые случаи взаимного расположения линий Стокса

Далее выясним, какие же взаимные расположения линий Стокса будут определять ребра спектрального графа. Вычислим матрицы монодромии для этих случаев и получим уравнения на точки спектра.

Теорема 3. Если Е принадлежит спектральному графу, то

1) существует конечная линия Стокса, соединяющая две точки поворота;

2) для любой чепочки канонических областей, инвариантной относительно сдвига $x \rightarrow x+2 \pi$, существует область из этой цепочки, содержащая конечную линию Стокса (т.е. существование конечной линии Стокса, которую нельзя обойти, на комплексной плоскости $x$, задает ребро спектрального графа на комплексной плоскости $E$ ).

ДокАЗАтельство. Рассмотрим расположения линий Стокса, когда нет конечной линии и когда она есть, но ее можно обойти. Вычислим для них матрицы монодромии и получим уравнения на точки спектра и на ребра спектрального графа. 


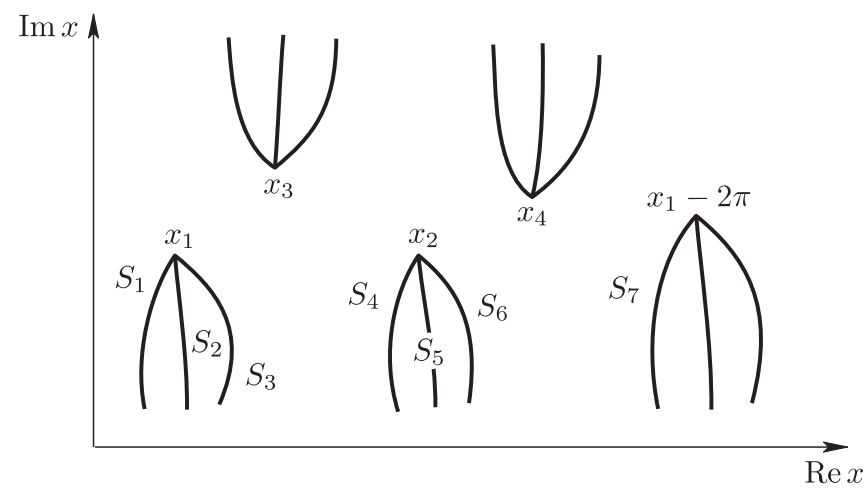

Рис. 6. Расположение линий Стокса, когда конечной линии нет

1. Случай, когда конечной линии нет (см. рис.6). Имеем четыре точки поворота, из каждой выходит по три линии Стокса, конечных линий нет. Необходимо совершить переход через период, т.е. через $2 \pi$. Для этого определим канонические области, по которым мы будем двигаться. Пусть с точкой $x_{1}$ и линией Стокса $S_{1}$ связана каноническая область $K_{1}$, с точкой $x_{1}$ и линией Стокса $S_{2}$ - область $K_{2}$, с точкой $x_{1}$ и линией Стокса $S_{3}$ - область $K_{3}$, с точкой $x_{2}$ и линией Стокса $S_{4}-$ область $K_{4}$, с точкой $x_{2}$ и линией Стокса $S_{5}$ - область $K_{5}$, с точкой $x_{2}$ и линией Стокса $S_{6}$ - область $K_{6}$ и, наконец, с точкой $x_{1}-2 \pi$ и линией Стокса $S_{1}-2 \pi=S_{7}-$ область $K_{7}$. Для того чтобы продолжить систему решений из канонической области $K_{7}$ в $K_{1}$, требуется построить матрицы перехода между областями и вычислить матрицу монодромии. Переход будет осуществляться следующим образом:

$$
\begin{aligned}
\left(x_{1}-2 \pi, S_{7}, K_{7}\right) \stackrel{M_{1}}{\longrightarrow}\left(x_{2}, S_{6}, K_{6}\right) \stackrel{M_{2}}{\longrightarrow}\left(x_{2}, S_{5}, K_{5}\right) \stackrel{M_{3}}{\longrightarrow}\left(x_{2}, S_{4}, K_{4}\right) \\
\stackrel{M_{4}}{\longrightarrow}\left(x_{1}, S_{3}, K_{3}\right) \stackrel{M_{5}}{\longrightarrow}\left(x_{1}, S_{2}, K_{2}\right) \stackrel{M_{6}}{\longrightarrow}\left(x_{1}, S_{1}, K_{1}\right) ;
\end{aligned}
$$

здесь $M_{i}$ - матрицы перехода, которые имеют следующий вид:

$$
M_{1}=\exp \left(\imath \xi_{0}\right)\left(\begin{array}{cc}
\exp \left(\frac{-\xi\left(x_{1}-2 \pi, x_{2}\right)}{\imath h}\right) & 0 \\
0 & \exp \left(\frac{\xi\left(x_{1}-2 \pi, x_{2}\right)}{\imath h}\right)
\end{array}\right)
$$

где

$$
\xi_{0}=\lim _{x \rightarrow x_{1}-2 \pi}\left(\arg (\imath V(x)-E)^{-1 / 4}\right)-\lim _{x \rightarrow x_{2}}\left(\arg (\imath V(x)-E)^{-1 / 4}\right), \quad \lambda=\frac{1}{\imath h},
$$

и

$$
\xi\left(x_{1}-2 \pi, x_{2}\right)=\int_{x_{1}-2 \pi}^{x_{2}} \sqrt{E-\imath V(x)} d x
$$

матрицы

$$
M_{2}=M_{3}=M_{5}=M_{6}=\exp \left(-\frac{\imath \pi}{6}\right)\left(\begin{array}{cc}
0 & 1 \\
1+O(h) & \imath(1+O(h))
\end{array}\right),
$$


и матрица

$$
M_{4}=\exp \left(\imath \xi_{0}\right)\left(\begin{array}{cc}
\exp \left(\frac{-\xi\left(x_{2}, x_{1}\right)}{\imath h}\right) & 0 \\
0 & \exp \left(\frac{\xi\left(x_{2}, x_{1}\right)}{\imath h}\right)
\end{array}\right)
$$

где

$$
\xi_{0}=\lim _{x \rightarrow x_{2}}\left(\arg (\imath V(x)-E)^{-1 / 4}\right)-\lim _{x \rightarrow x_{1}}\left(\arg (\imath V(x)-E)^{-1 / 4}\right) .
$$

Для вычисления матрицы монодромии перемножим матрицы перехода $M=$ $M_{1} M_{2} M_{3} M_{4} M_{5} M_{6}$ и получим

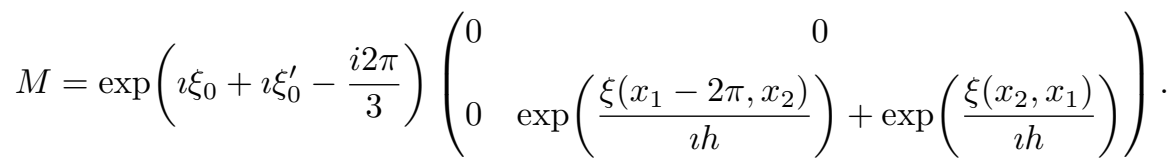

Отсюда получаем, что уравнение на точку спектра и на ребро спектрального графа $\operatorname{Tr} M=\operatorname{det} M+1$ в данном случае имеет вид

$$
\exp \left(\imath \xi_{0}+\imath \xi_{0}^{\prime}-\frac{\imath 2 \pi}{3}\right)\left(\exp \left(\frac{\xi\left(x_{1}-2 \pi, x_{2}\right)}{\imath h}\right)+\exp \left(\frac{\xi\left(x_{2}, x_{1}\right)}{\imath h}\right)\right)=1+O(h) .
$$

Так как ветвь выбирается следующим образом:

$$
\operatorname{Re} \xi\left(x_{2}, x_{1}\right)>0, \quad \operatorname{Re} \xi\left(x_{1}-2 \pi, x_{2}\right)>0,
$$

получаем, что при $h \rightarrow 0+0$ выполнено $|\operatorname{Tr} M| \rightarrow \infty$, т.е. такие точки $E$ не принадлежат спектру; другими словами, этот случай ничего не вносит в асимптотику спектра искомого оператора. Аналогично рассматриваются остальные случаи, в которых нет конечной линии Стокса.

2. Случай, когда конечная линия существует, но ее можно обойти. Ситуация здесь похожа на рассмотренную в первом пункте (см. рис. 7).

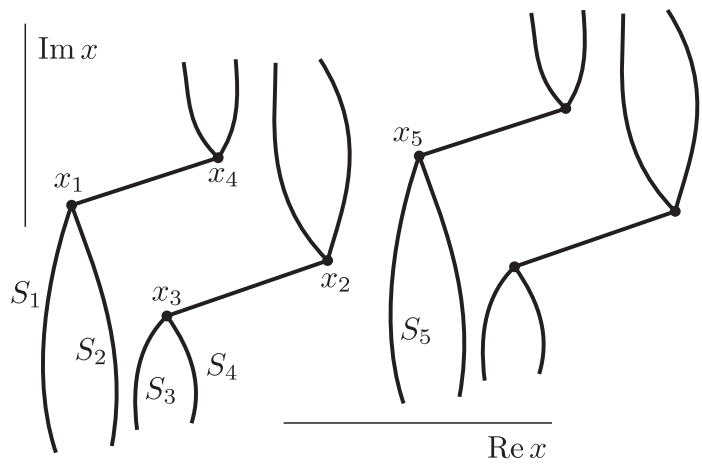

Рис. 7. Расположение линий Стокса, когда конечную линию можно обойти

Определим канонические области и путь, по которому будем двигаться:

$$
\left(x_{5}, S_{5}, K_{5}\right) \stackrel{M_{1}}{\longrightarrow}\left(x_{3}, S_{4}, K_{4}\right) \stackrel{M_{2}}{\longrightarrow}\left(x_{3}, S_{3}, K_{3}\right) \stackrel{M_{3}}{\longrightarrow}\left(x_{1}, S_{2}, K_{2}\right) \stackrel{M_{4}}{\longrightarrow}\left(x_{1}, S_{1}, K_{1}\right),
$$


где $x_{5}+2 \pi=x_{1}, K_{5}+2 \pi=K_{1}$. Матрицы перехода имеют следующий вид:

$$
M_{2}=M_{4}=\exp \left(-\frac{\imath \pi}{6}\right)\left(\begin{array}{cc}
0 & 1 \\
1+O(h) & \imath(1+O(h))
\end{array}\right),
$$

и

$$
\begin{aligned}
& M_{1}=\exp \left(\imath \xi_{0}\right)\left(\begin{array}{cc}
\exp \left(\frac{-\xi\left(x_{5}, x_{3}\right)}{\imath h}\right) & 0 \\
0 & \exp \left(\frac{\xi\left(x_{5}, x_{3}\right)}{\imath h}\right)
\end{array}\right), \\
& M_{3}=\exp \left(\imath \xi_{0}\right)\left(\begin{array}{cc}
\exp \left(\frac{-\xi\left(x_{3}, x_{1}\right)}{\imath h}\right) & 0 \\
0 & \exp \left(\frac{\xi\left(x_{3}, x_{1}\right)}{\imath h}\right)
\end{array}\right) .
\end{aligned}
$$

В итоге получаем матрицу монодромии

$$
\begin{aligned}
& \exp \left(\imath\left(\xi_{0}+\xi_{0}^{\prime}-\frac{\pi}{3}\right)\right) \\
& \times\left(\begin{array}{cc}
\exp \left(\frac{-\xi\left(x_{5}, x_{3}\right)+\xi\left(x_{3}, x_{1}\right)}{\imath h}\right) & \imath \exp \left(\frac{-\xi\left(x_{5}, x_{3}\right)+\xi\left(x_{3}, x_{1}\right)}{\imath h}\right) \\
\imath \exp \left(\frac{\xi\left(x_{5}, x_{3}\right)+\xi\left(x_{3}, x_{1}\right)}{\imath h}\right) & \exp \left(\frac{\xi\left(x_{5}, x_{3}\right)-\xi\left(x_{3}, x_{1}\right)}{\imath h}\right)-\exp \left(\frac{\xi\left(x_{5}, x_{3}\right)+\xi\left(x_{3}, x_{1}\right)}{\imath h}\right)
\end{array}\right) .
\end{aligned}
$$

Если $E$ принадлежит спектру, то

$$
\cos \left(\frac{\xi\left(x_{5}, x_{3}\right)-\xi\left(x_{3}, x_{1}\right)}{\imath h}\right)=\exp \left(\frac{\xi\left(x_{5}, x_{3}\right)+\xi\left(x_{3}, x_{1}\right)}{\imath h}\right)+2+O(h) .
$$

Замечая, что ветвь выбрана так же, как и в предыдущем случае $\left(\operatorname{Re} \xi\left(x_{5}, x_{3}\right)>0\right.$, $\left.\operatorname{Re} \xi\left(x_{3}, x_{1}\right)>0\right)$, получаем следующий предел:

$$
\exp \left(\frac{\xi\left(x_{5}, x_{3}\right)+\xi\left(x_{3}, x_{1}\right)}{\imath h}\right) \rightarrow \infty .
$$

Значит, этот случай также не вносит вклад в асимптотику спектра.

Аналогично рассматриваются остальные случаи, в которых можно обойти конечную линию Стокса. Теорема 3 доказана.

Лемма 3. Существует всего семь возможных расположений линий Стокса, которые могут вносить вклад в спектр.

ДОКАЗАТЕЛЬСТВО следует из свойств 1)-8) и из предыдущей теоремы прямым перебором. На рис. 8 приведены эти семь топологических случаев расположения линий Стокса. В следующем списке указано, какие точки поворота соединяются между собой в этих случаях (см. рис. 8):
a) $z_{j} \rightarrow-z_{j}$
b) $z_{j} \rightarrow-z_{j}+2 \pi$
c) $z_{j} \rightarrow-z_{j}+2 \pi$
d) $z_{i} \rightarrow z_{j}$;
e) $z_{i} \rightarrow z_{j}-2 \pi$;
f) $z_{i} \rightarrow z_{j}+2 \pi$;
g) $z_{i} \rightarrow z_{j} \rightarrow z_{i}+2 \pi \rightarrow z_{j}+2 \pi$. 


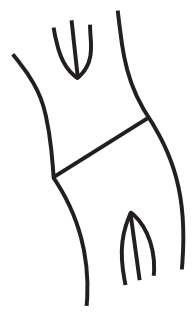

a)

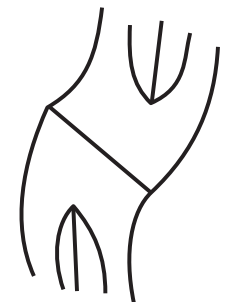

b)

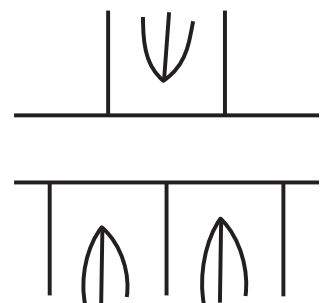

c)

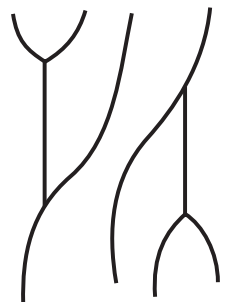

d)

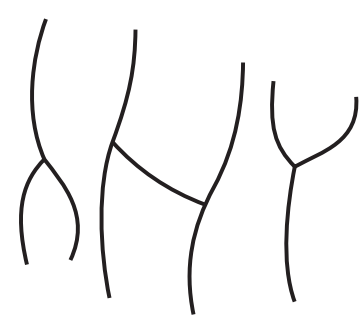

e)

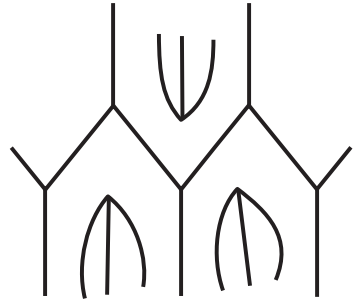

f)

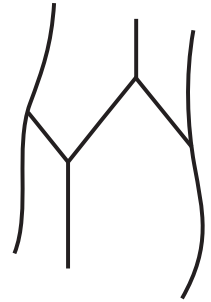

g)

Рис. 8. Семь топологических случаев взаимного расположения линий

9. Матрицы монодромии и уравнения на точки спектра для семи топологических случаев взаимного расположения линий Стокса. Топологический случай 1) (см. рис. 9). Здесь $S_{1}$ - линия Стокса, выходящая из точки поворота $z_{1}$ вверх, $S_{3}$ - линия Стокса, выходящая из точки поворота $z_{2}, S_{2}$ соединяет $z_{1}$ и $z_{2}, z_{2}=z_{3}+2 \pi$.
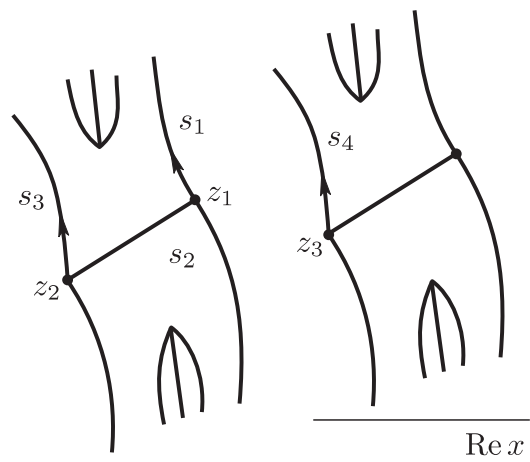

$\operatorname{Im} x$

Рис. 9. Топологический случай 1)

Каждую из линий Стокса можно включить в соответствующую каноническую область.

Пусть у точки $z_{3}$ и линией Стокса, выходящей из нее вертикально вверх каноническая область $K_{1}$, тогда у точки поворота $z_{1}$ и линией Стокса, выходящей из нее вертикально вверх, каноническая область $K_{2}$. У $z_{1}$ и линии Стокса $S_{2}$ каноническая область $K_{3}$, у $z_{2}$ и $S_{2}$ - каноническая область $K_{4}$ и, наконец, у $z_{2}$ и $S_{3}$ - каноническая 
область $K_{5}=K_{1}+2 \pi$. Для того чтобы продолжить систему решений из области $K_{1}$ в область $K_{5}$, требуется построить матрицы перехода $M_{k}, k=1,2,3,4$ :

$$
\left(z_{3}, S_{4}, K_{1}\right) \stackrel{M_{1}}{\longrightarrow}\left(z_{1}, S_{1}, K_{2}\right) \stackrel{M_{2}}{\longrightarrow}\left(z_{1}, S_{2}, K_{3}\right) \stackrel{M_{3}}{\longrightarrow}\left(z_{2}, S_{2}, K_{4}\right) \stackrel{M_{4}}{\longrightarrow}\left(z_{2}, S_{3}, K_{1}+2 \pi\right) .
$$

Матрицы $M_{k}, k=1,2,3,4$, имеют следующий вид:

$$
M_{2}=M_{4}=\exp \left(-\frac{\imath \pi}{6}\right)\left(\begin{array}{ll}
0 & 1 \\
1 & \imath
\end{array}\right)
$$

И

$$
\begin{aligned}
& M_{1}=\exp \left(\imath \xi_{0}\right)\left(\begin{array}{cc}
\exp \left(\frac{-\xi\left(z_{3}, z_{1}\right)}{\imath h}\right) & 0 \\
0 & \exp \left(\frac{\xi\left(z_{3}, z_{1}\right)}{\imath h}\right)
\end{array}\right), \\
& M_{3}=\exp \left(\imath \widetilde{\xi}_{0}\right)\left(\begin{array}{cc}
0 & \exp \left(\frac{-\xi\left(z_{1}, z_{2}\right)}{\imath h}\right) \\
\exp \left(\frac{\xi\left(z_{1}, z_{2}\right)}{\imath h}\right) & 0
\end{array}\right),
\end{aligned}
$$

где

$$
\begin{gathered}
\int \sqrt{\imath V(x)-E} d x=\xi, \\
\xi_{0}=\lim _{x \rightarrow z_{3}}\left(\arg (\imath V(x)-E)^{-1 / 4}\right)-\lim _{x \rightarrow z_{1}}\left(\arg (\imath V(x)-E)^{-1 / 4}\right)=-\frac{\imath \pi}{6} .
\end{gathered}
$$

Имеем $M=M_{1} M_{2} M_{3} M_{4}$. Получаем матрицу монодромии

$$
\begin{aligned}
& \exp \left(\imath\left(\xi_{0}+\widetilde{\xi}_{0}-\frac{\pi}{3}\right)\right) \\
& \times\left(\begin{array}{c}
0 \\
\exp \left(\frac{\xi\left(z_{3}, z_{1}\right)-\xi\left(z_{1}, z_{2}\right)}{\imath h}\right) \quad \imath\left(\exp \left(\frac{-\xi\left(z_{3}, z_{1}\right)+\xi\left(z_{1}, z_{2}\right)}{\imath h}\right)+\exp \left(\frac{\xi\left(z_{3}, z_{1}\right)-\xi\left(z_{1}, z_{2}\right)}{\imath h}\right)\right)
\end{array}\right) .
\end{aligned}
$$

Запишем условие принадлежности $E$ спектру $(\operatorname{tr} M=\operatorname{det} M+1)$ :

$$
\begin{aligned}
& \exp \left(\imath\left(\xi_{0}+\widetilde{\xi}_{0}-\frac{\pi}{3}\right)\right) \imath\left(\exp \left(\frac{-\xi\left(z_{3}, z_{1}\right)+\xi\left(z_{1}, z_{2}\right)}{\imath h}\right)+\exp \left(\frac{\xi\left(z_{3}, z_{1}\right)-\xi\left(z_{1}, z_{2}\right)}{\imath h}\right)\right) \\
& \quad=-\exp \left(2 \imath\left(\xi_{0}+\widetilde{\xi}_{0}-\frac{\pi}{3}\right)\right)+1 .
\end{aligned}
$$

Поскольку $\xi_{0}+\widetilde{\xi}_{0}=\pi / 3$, отсюда вытекает равенство

$$
2 \exp \left(\frac{\xi\left(z_{3}, z_{1}\right)}{\imath h}\right) \cos \left(\frac{\xi\left(z_{1}, z_{2}\right)}{\imath h}\right)=2 .
$$

Так как ветвь выбрана так, что $\operatorname{Im} \xi\left(z_{0}, z\right)>0$ (где $z_{0}$ - точка поворота), то

$$
\cos \left(\frac{\xi\left(z_{1}, z_{2}\right)}{\imath h}\right)=\exp \left(\frac{-\xi\left(z_{3}, z_{1}\right)}{\imath h}\right), \quad \exp \left(\frac{-\xi\left(z_{3}, z_{1}\right)}{\imath h}\right) \rightarrow 0 .
$$


Отсюда получаем следующее уравнение на точки спектра и на ребро спектрального графа:

$$
\int_{z_{1}}^{z_{2}} \sqrt{E-\imath V(x)} d x=\imath h\left(\frac{\pi}{2}+\pi n\right), \quad n=1,2, \ldots
$$

Из этого условия следует, что $\operatorname{Re} \xi\left(z_{1}, z_{2}\right)=0$; именно этим уравнением определяется ребро спектрального графа на комплексной плоскости $E$.

Точки спектра будут определяться из уравнения

$$
\operatorname{Im} \int_{z_{1}}^{z_{2}} \sqrt{E-\imath V(x)} d x=h\left(\frac{\pi}{2}+\pi n\right), \quad n=1,2, \ldots .
$$

Топологический случай 2). Этот случай аналогичен предыдущему, с тем лишь различием, что в уравнении на точки спектра интеграл будет между другими точками поворота, т.е.

$$
\int_{z_{1}}^{z_{2}+2 \pi} \sqrt{E-\imath V(x)} d x=\imath h\left(\frac{\pi}{2}+\pi n\right), \quad n=1,2, \ldots
$$

В этом случае ребро спектрального графа на комплексной плоскости $E$ определяется уравнением $\operatorname{Re} \xi\left(z_{1}, z_{2}+2 \pi\right)=0$; это ребро будет отличаться от предыдущего случая.
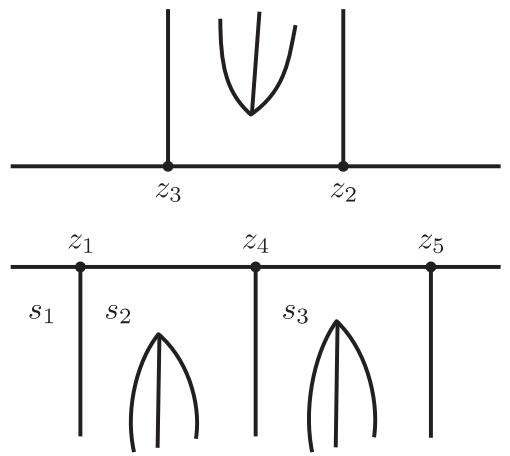

Рис. 10. Топологический случай 3)

Топологический случай 3) (см. рис. 10). Обозначим $z_{1}, z_{2}, z_{3}, z_{4}=z_{1}-2 \pi$ - точки поворота, $S_{1}$ - линия Стокса, соединяющая точки $z_{1}$ и $z_{1}+2 \pi, S_{2}$ - линия Стокса, соединяющая точки $z_{1}$ и $z_{4}, S_{3}$ - линия Стокса, соединяющая точки $z_{4}$ и $z_{5}$.

Каждой точке и линии Стокса соответствует каноническая область. Пусть точке $z_{4}$ и линии Стокса $S_{2}$ соответствует каноническая область $K_{1}$, точке $z_{1}$ и линии Стокса $S_{2}$ - область $K_{2}$, точке $z_{1}$ и линии Стокса $S_{1}$ - область $K_{3}\left(K_{3}=K_{1}+2 \pi\right)$. Для того чтобы продолжить систему решений из области $K_{1}$ в область $K_{3}$, требуется построить матрицы перехода $M_{k}, k=1,2,3,4$ :

$$
\left(z_{4}, S_{2}, K_{1}\right) \stackrel{M_{1}}{\longrightarrow}\left(z_{1}, S_{2}, K_{2}\right) \stackrel{M_{2}}{\longrightarrow}\left(z_{1}, S_{1}, K_{3}\right)
$$


Как и в предыдущем случае, получаем матрицу монодромии

$$
M=\exp \left(\imath\left(\tau_{0}-\frac{\pi}{6}\right)\right)\left(\begin{array}{cc}
0 & \exp \left(\frac{\tau_{1}}{\imath h}\right) \\
\exp \left(\frac{-\tau}{\imath h}\right) & \imath\left(\exp \left(\frac{\tau_{1}}{\imath h}\right)+\exp \left(\frac{-\tau}{\imath h}\right)\right)
\end{array}\right)
$$

где

$$
\begin{gathered}
\int \sqrt{\imath V(x)-E} d x=\xi, \quad \int_{z_{1}}^{z_{4}} \sqrt{\imath V(x)-E} d x=\tau, \\
\tau_{0}=\lim _{x \rightarrow z_{4}}\left(\arg (\imath V(x)-E)^{-1 / 4}\right)-\lim _{x \rightarrow z_{1}}\left(\arg (\imath V(x)-E)^{-1 / 4}\right)=-\frac{\imath \pi}{6} .
\end{gathered}
$$

Так же, как и в предыдущем случае, получим уравнение на точки спектра и на ребро спектрального графа. Имеем

$$
\tau=\imath h 2 \pi n, \quad n=0,1,2, \ldots .
$$

Так как

$$
\tau=\int_{0}^{2 \pi} \sqrt{\imath V(x)-E} d x
$$

$\left(z_{4}+2 \pi=z_{1}\right)$, получаем такое уравнение

$$
\int_{0}^{2 \pi} \sqrt{\imath V(x)-E} d x=\imath h 2 \pi n .
$$

Отсюда,

$$
\operatorname{Re} \int_{0}^{2 \pi} \sqrt{\imath V(x)-E} d x=0
$$

это условие определяет другое ребро спектрального графа.

Топологический случай 4) (см. рис. 11). Точно так же, как и в предыдущих случаях, получаем матрицу монодромии

$$
\exp \left(\imath\left(\tau_{0}+\tau_{0}^{\prime}-\frac{\pi}{3}\right)\right)\left(\begin{array}{cc}
\exp \left(\frac{-\tau_{1}+\tau_{2}+\tau_{3}}{\imath h}\right) & \exp \left(\frac{\tau_{1}-\tau_{2}-\tau_{3}}{\imath h}\right) \\
\imath \exp \left(\frac{-\tau_{1}+\tau_{2}+\tau_{3}}{\imath h}\right) & \exp \left(\frac{\tau_{1}-\tau_{2}-\tau_{3}}{\imath h}\right)-\exp \left(\frac{\tau_{1}-\tau_{2}-\tau_{3}}{\imath h}\right)
\end{array}\right),
$$

где

$$
\int_{z_{1}}^{z_{4}} \sqrt{\imath V(x)-E} d x=\tau_{1}, \quad \int_{z_{4}}^{z_{6}} \sqrt{\imath V(x)-E} d x=\tau_{2}, \quad \int_{z_{6}}^{z_{5}} \sqrt{\imath V(x)-E} d x=\tau_{3},
$$

и уравнение на точки спектра и на ребро спектрального графа:

$$
\int_{z_{6}}^{z_{5}} \sqrt{\imath V(x)-E} d x=\imath h\left(\frac{\pi}{2}+\pi n\right) .
$$

Ребро спектрального графа, таким образом, выглядит так:

$$
\operatorname{Re} \int_{z_{6}}^{z_{5}} \sqrt{\imath V(x)-E} d x=0 .
$$




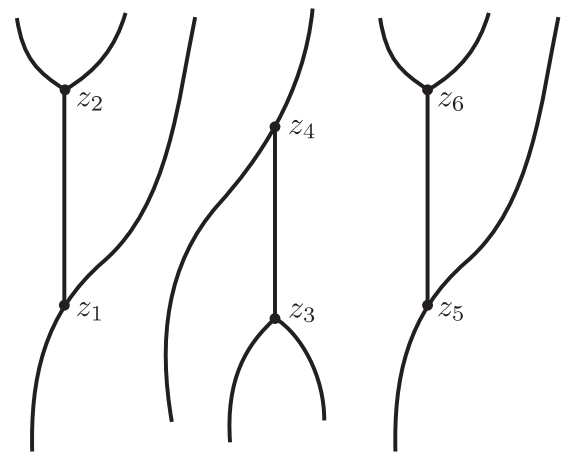

Рис. 11. Топологический случай 4)

Остальные топологические случаи рассматриваются аналогично. Приведем результаты - уравнения на точки спектра и на ребра графа.

Топологический случай 5) (см. рис. 12). Уравнение на точки спектра и ребро графа имеет следующий вид:

$$
\int_{z_{4}}^{z_{3}} \sqrt{\imath V(x)-E} d x=\imath h\left(\frac{\pi}{2}+\pi n\right) .
$$

Ребро спектрального графа в этом случае задается уравнением

$$
\operatorname{Re} \int_{z_{4}}^{z_{3}} \sqrt{\imath V(x)-E} d x=0 .
$$

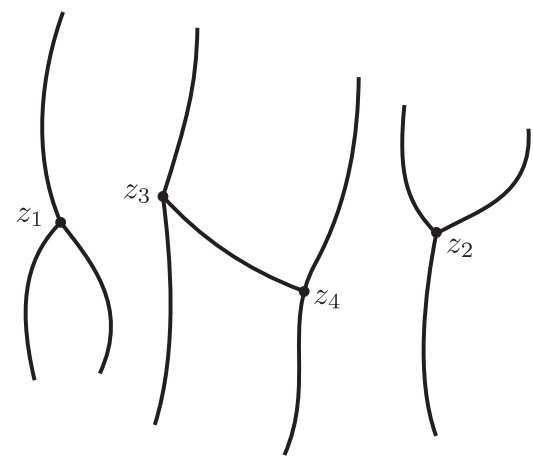

Рис. 12. Топологический случай 5)

Топологический случай 6) (см. рис. 13). Уравнение на точки спектра и ребро графа имеет следующий вид:

$$
\int_{0}^{2 \pi} \sqrt{\imath V(x)-E} d x=\imath h 2 \pi n .
$$

Ребро спектрального графа определяется уравнением

$$
\operatorname{Re} \int_{0}^{2 \pi} \sqrt{\imath V(x)-E} d x=0 .
$$




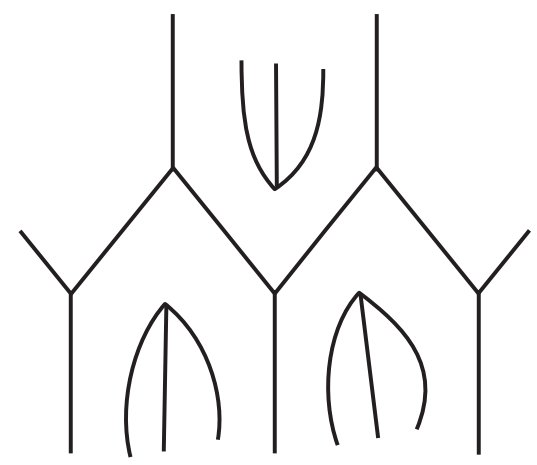

Рис. 13. Топологический случай 6)

Топологический случай 7) (см. рис. 14). Уравнение на точки спектра и ребро графа имеет следующий вид:

$$
\int_{0}^{2 \pi} \sqrt{\imath V(x)-E} d x=\imath h 2 \pi n .
$$

Ребро спектрального графа:

$$
\operatorname{Re} \int_{0}^{2 \pi} \sqrt{\imath V(x)-E} d x=0 .
$$

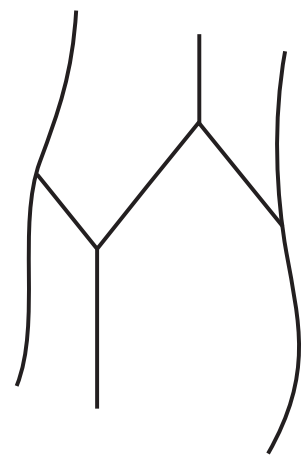

Рис. 14. Топологический случай 7)

Таким образом, каждый случай, который вносит вклад в спектр, задает ребро спектрального графа на комплексной плоскости $E$. В это условие входит интеграл между точками поворота, которые соединены линией Стокса, причем эту линию Стокса нельзя обойти. То есть существование конечной линии Стокса, которую нельзя обойти, на комплексной плоскости $x$, задает ребро спектрального графа на комплексной плоскости $E$. Теорема 1 доказана.

ЗАмечАниЕ 2. Таким образом, каждый из 7 топологических случаев расположения линий Стокса определяет свою серию собственных значений и свое ребро спектрального графа. Ребра определяются равенствами (2.2), а точки спектра вычисляются по формулам (2.1). 
10. Описание спектрального графа. Спектральный граф (см. рис. 1) получен с помощью компьютера по уравнениям (2.2). Различным ребрам соответствуют различные топологические случаи расположения линий Стокса; именно:

- ребру 1 соответствует топологический случай под номером 1 ;

- ребру 2 соответствует топологический случай под номером 4;

- ребру 3 соответствует топологический случай под номером 5 ;

- ребру 6 соответствует топологический случай под номером 3 ;

- ребру 4 соответствует топологический случай под номером 5 ;

- ребру под номером 5 соответствует топологический случай под номером 3;

- точке соединения ребер 1 и 5 соответствует топологический случай 6);

- точке соединения ребер 1 и 2 соответствует топологический случай 7).

Ниже доказаны некоторые свойства спектрального графа.

\section{1. Некоторые факты относительно ребер спектрального графа.}

УтвеРЖДЕНИЕ 1. Если $E \in \mathbb{C} \backslash \imath(-9 / 8,2)$ и выполняется равенство

$$
I(E)=\operatorname{Re} \int_{0}^{2 \pi} \sqrt{\imath(\cos x+\cos 2 x)-E} d x=0,
$$

mо $\operatorname{Re} E>0, \operatorname{Im} E<0$. (Это означает, что ребра под номерами 5 и 6 лежат ниже оси $\operatorname{Re} E$.)

ДокАзАтЕЛьство. Будем анализировать функцию такого вида:

$$
I(E)=\operatorname{Re} \int_{0}^{2 \pi} \sqrt{\imath(\cos x+\cos 2 x)-E} d x=2 \operatorname{Re} \int_{0}^{\pi} \sqrt{\imath(\cos x+\cos 2 x)-E} d x .
$$

Рассмотрим $E=\operatorname{Re} E<0$. Следовательно, нулей подынтегрального выражения нет. Знак $\operatorname{Re} \sqrt{\imath(\cos x+\cos 2 x)-E}$ постоянен, следовательно, отличен от нуля на всем пути интегрирования и $I(E) \neq 0$. А значит, мы пришли к противоречию и $\operatorname{Re} E>0$.

Рассмотрим $E$ с положительной действительной частью. Получаем

$$
\operatorname{Re}(\imath(\cos x+\cos 2 x)-E)=-\operatorname{Re} E<0
$$

для любого $x$, принадлежащего отрезку $(0, \pi)$. То есть образ пути интегрирования под действием функции $\imath(\cos x+\cos 2 x)-E$ не переходит через луч $[0,+\infty)$. Следовательно, можно зафиксировать ветвь корня с разрезом вдоль этого луча. Выберем следующую ветвь:

$$
\sqrt{ }: \mathbb{C} \backslash[0,+\infty) \rightarrow\{z \in \mathbb{C} \mid \operatorname{Im} z>0\}
$$

тогда

$$
\begin{aligned}
\frac{\partial \operatorname{Re} \sqrt{\imath(\cos x+\cos 2 x)-E}}{\partial \operatorname{Im} E} & =-\operatorname{Re} \frac{\imath}{2 \sqrt{\imath(\cos x+\cos 2 x-E}} \\
& =-\operatorname{Im} \frac{1}{2 \sqrt{\imath(\cos x+\cos 2 x)-E}}>0 .
\end{aligned}
$$

Следовательно, подынтегральная функция возрастает на всем пути интегрирования для любого $x \in(0, \pi)$. А значит, и сам интеграл строго монотонно возрастает по $\operatorname{Im} E$ 
при любом фиксированном Re $E$. И при любом фиксированном $\operatorname{Re} E>0$ уравнение $I(E)=0$ имеет не более одного решения. Разложим подынтегральную функцию в ряд Тейлора:

$$
\begin{aligned}
& \sqrt{\imath(\cos x+\cos 2 x)-E} \\
& \quad=\sqrt{-E}\left(1-\frac{\imath(\cos x+\cos 2 x)}{2 E}+\frac{(\cos x+\cos 2 x)^{2}}{8 E^{2}}+\frac{\imath(\cos x+\cos 2 x)^{3}}{16 E^{3}}+\cdots\right)
\end{aligned}
$$

и проинтегрируем:

$$
\operatorname{Re} \int_{0}^{2 \pi} \sqrt{\imath(\cos x+\cos 2 x)-E} d x=\operatorname{Re}\left(2 \sqrt{-E}\left(\left(1+\frac{E^{2}}{8}\right) \pi+\frac{3 \pi \imath}{32 E^{3}}\right)\right) .
$$

Рассмотрим случай $\mathrm{Im}=0, \operatorname{Re} E>0$. Имеем

$$
I(E)=-\frac{3 \pi}{16 E^{2} \sqrt{E}}<0
$$

при любом фиксированном $\operatorname{Re} E$. Отсюда следует, что кривая $I(E)=0$ будет находиться ниже оси Re E. Утверждение доказано.

УтвеРЖДЕНИЕ 2. Уравнение относителъно Е имеет вид

$$
J(E)=\operatorname{Im} \int_{-x_{j}}^{x_{j}} \sqrt{\imath(\cos x+\cos 2 x)-E} d x=0,
$$

¿əe

$$
x_{1,2}= \pm \operatorname{Arccos}\left(\frac{1}{4}(-1-\sqrt{9-8 \imath E})\right), \quad x_{3,4}= \pm \operatorname{Arccos}\left(\frac{1}{4}(-1+\sqrt{9-8 \imath E})\right),
$$

при условии, что

$$
I(E)=\operatorname{Re} \int_{0}^{2 \pi} \sqrt{\imath(\cos x+\cos 2 x)-E} d x=0
$$

имеет ровно одно решение. (Это утверждение означает, что ребра под номерами 1 и 4 пересекают ребра 5 и 6 соответственно толъко в одной точке.)

ДокАЗАТЕЛЬство. Как и в предыдущем утверждении, зафиксируем ветвь корня:

$$
\sqrt{ }: \mathbb{C} \backslash[0,+\infty) \rightarrow\{z \in \mathbb{C} \mid \operatorname{Im} z>0\}
$$

Перепишем интеграл $J(E)$ в виде интеграла по действительной переменной $r$. Имеем

$$
\begin{aligned}
\operatorname{Re} \int_{-x_{j}(E)}^{x_{j}(E)} \sqrt{\imath(\cos x+\cos 2 x)-E} d x & =2 \operatorname{Re} \int_{0}^{x_{j}(E)} \sqrt{\imath(\cos x+\cos 2 x)-E} d x \\
& =2 \operatorname{Re} \int_{0}^{1} x_{j} \sqrt{\imath\left(\cos x_{j} r+\cos 2 x_{j} r\right)-E} d r .
\end{aligned}
$$

Вычислим производную подынтегрального выражения по параметру $E$ :

$$
\frac{\partial \operatorname{Re}\left(x_{j} \sqrt{\imath\left(\cos x_{j} r+\cos 2 x_{j} r\right)-E}\right)}{\partial \operatorname{Im} E}=-\operatorname{Re} \frac{x_{j} \imath}{2 \sqrt{\imath\left(\cos x_{j} r+\cos 2 x_{j} r\right)-E}}>0 .
$$


Интеграл строго монотонно возрастает по параметру $\operatorname{Im} E$ при любом фиксированном $\operatorname{Re} E>0$. И при любом фиксированном $\operatorname{Re} E>0$ уравнение $J(E)=0$ имеет не более одного решения. Отсюда следует, что при любом фиксированном $\operatorname{Re} E>0$ уравнение $I(E)=0$ имеет не более одного решения. Следовательно, система уравнений $I(E)=0$ и $J(E)=0$ имеет не более одного решения. Утверждение доказано.

12. Условие квантования на римановой поверхности. Оказывается, асимптотика спектра оператора $\widehat{H}$ выражается через интегралы от голоморфной формы $p d x$ по циклам на римановой поверхности, задаваемой уравнением

$$
p^{2}+\imath(\cos x+\cos 2 x)=E .
$$

Эта поверхность получается путем склейки двух цилиндров $(x \in \mathbb{C} \bmod 2 \pi)$ вдоль одного конечного разреза (соединяющего две точки поворота) и двух бесконечных разрезов (соединяющих оставшиеся точки с бесконечностями); она гомеоморфна сфере с двумя ручками и двумя проколами. Условия, определяющие спектр, имеют вид

$$
\frac{1}{2 \pi h} \int_{\gamma_{j}} p d x=n_{j}+\frac{\mu_{j}}{4},
$$

где $\gamma_{j}$ - один из циклов римановой поверхности, изображенных на рис. $15, \mu_{j} \in$ $\{0,2\}$. Отметим, что для того чтобы точка $E$ приближала точку спектра, достаточно выполнения условия квантования на одном цикле римановой поверхности (а не на всех, как в вещественной теории; см., например, [1]). Условие квантования, соответствующее каждому циклу, определяет серию собственных значений, лежащую на определенном ребре спектрального графа. Циклу под номером 1 соответствует ребро 5 спектрального графа, циклу 2 - ребро 1 , циклу 5 - ребро 2 , циклу 4 - ребро 4 и циклу 3 - ребро 3.

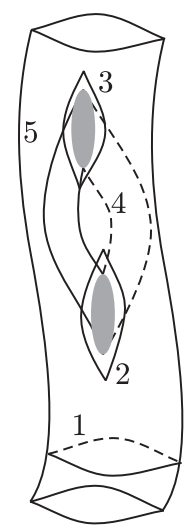

Рис. 15. Риманова поверхность

\section{СПИСОК ЦИТИРОВАННОЙ ЛИТЕРАТУРЫ}

[1] В. П. Маслов, Теория возмущений и асимптотические методы, Изд-во Моск. ун-та, M., 1965. 
[2] E. B. Davies, "Pseudospectra of differential operators", J. Operator Theory, 43:2 (2000), 243-262.

[3] P. G. Drazin, W. H. Reid, Hydrodynamic Stability, Cambridge Monogr. Mech. Appl. Math., Cambridge Univ. Press, Cambridge, 1981.

[4] Я. Б. Зельдович, А. А. Рузмайкин, "Гидромагнитное динамо как источник планетарного, солнечного и галактического магнетизма", УФН, 152:2 (1987), 263-284.

[5] С.А. Степин, А.А. Аржанов, "Квазиклассические спектральные асимптотики и явление Стокса для уравнения Вебера", Докл. РАН, 378:1 (2001), 18-21.

[6] С.В.Гальцев, А. И. Шафаревич, "Спектр и псевдоспектр несамосопряженного оператора Шрёдингера с периодическими коэффициентами”, Матем. заметки, 80:3 (2006), 356-366.

[7] С. В. Гальцев, А. И. Шафаревич, "Квантованные римановы поверхности и квазиклассические спектральные серии для несамосопряженного оператора Шредингера с периодическими коэффициентами", ТМФ, 148:2 (2006), 206-226.

[8] А. В. Дьяченко, А. А. Шкаликов, "О модельной задаче для уравнения Орра-Зоммерфельда с линейным профилем", Функи. анализ и его прил., 36:3 (2002), 71-75.

[9] С.А. Степин, "Несамосопряженные сингулярные возмущения: модель перехода от дискретного спектра к непрерывному", УМН, 50:6 (1995), 219-220.

[10] С.А. Степин, В.А. Титов, “О возмущении кратного собственного значения", УМН, 60:1 (2005), 155-156.

[11] С. Н. Туманов, А. А. Шкаликов, "О предельном поведении спектра модельной задачи для уравнения Орра-Зоммерфельда с профилем Пуазейля", Изв. РАН. Сер. матем., 66:4 (2002), 177-204.

[12] А. А. Шкаликов, "О предельном поведении спектра при больших значениях параметра одной модельной задачи", Матем. заметки, 62:6 (1997), 950-953.

[13] L. N. Trefethen, "Pseudospectra of linear operators", ISIAM 95, Proceedings of the Third international congress on industrial and applied mathematics (Hamburg, 1995), Math. Res., 87, Akademie Verlag, Berlin, 1996, 401-434.

[14] М.В. Федорюк, Асимптотические методы для линейных обыкновенных дифференицальных уравнений, Справочная математическая библиотека, Наука, М., 1983.

[15] М. А. Евграфов, М. В. Федорюк, “Асимптотика решений уравнения $w^{\prime \prime}(z)-p(z, \lambda) \times$ $w(z)=0$ при $\lambda \rightarrow \infty$ в комплексной плотности $z^{\prime \prime}$, УMH, 21:1 (1966), 3-50.

А. И. Есина

Московский физико-технический институт

(государственный университет)

E-mail: esina_anna@list.ru

\section{А. И. Шафаревич}

Московский государственный университет

им. М. В. Ломоносова,

Институт проблем механики им. А. Ю. Ишлинского РАН
Поступило

25.11.2009 